
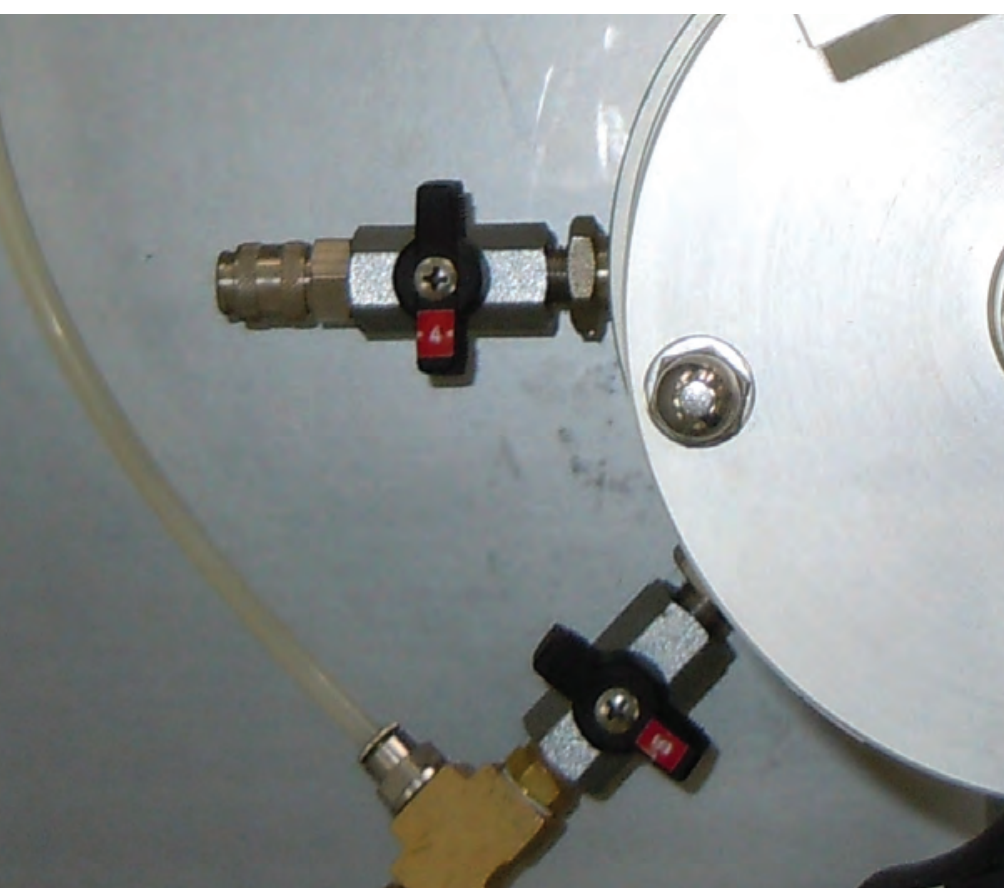

\title{
Ensayos triaxiales para suelos
}

Fecha de recepción: 29 de agosto del 2011

Fecha de aceptación: 29 de setiembre del 2011

\section{Resumen}

En este documento, se presenta una breve explicación de la teoría del esfuerzo cortante, una descripción general de los principales tipos de ensayos triaxiales que se realizan con mayor frecuencia en un laboratorio de geotecnia, así como una detallada enumeración de los diferentes componentes que conforman el equipo de ensayos triaxial adquirido por el laboratorio de geotecnia del LanammeUCR. Finalmente se presenta el montaje y algunos de los resultados producto de la ejecución de un ensayo UU.

\section{Palabras clave:}

Suelos, máquina triaxial, resistencia al corte, cohesión, ángulo de fricción.
Ing. Oscar Valerio Salas, LanammeUCR

\section{Introducción}

El esfuerzo cortante en los suelos es el aspecto más importante de la ingeniería geotécnica. La capacidad de soporte de cimentaciones superficiales como profundas, la estabilidad de los taludes y el diseño de muros o paredes de retención, llevan implícito el valor de la resistencia al esfuerzo cortante. Desde otro punto de vista, el diseño de los pavimentos, se ve influenciado de una forma indirecta por la resistencia al

\begin{abstract}
This document presents a brief explanation of the shear strength theory, an overview of the main types of triaxial tests that are performed more frequently in a geotechnical laboratory and a detailed listing of the different components that make up the triaxial test equipment acquired by the LanammeUCR Geotechnical Laboratory. Finally it presents the assembly and some of the results from the execution of a UU triaxial test.
\end{abstract}

\section{Keywords:}

Soils, triaxial apparatus, shear strength, cohesion, friction angle.

cortante de los suelos, ya sea en el análisis de la estabilidad de un talud o en el diseño de los muros de retención y de forma directa, a través del diseño de las fundaciones que soportan el pavimento, específicamente, en la subrasante. Por consecuencia, tanto las estructuras como los taludes deben ser estables y seguros frente a un colapso total, cuando éstos sean sometidos a una máxima aplicación de cargas. [13] 
El esfuerzo cortante de un suelo se ha definido como la última o máxima resistencia que el suelo puede soportar. Específicamente, se ha expresado como la resistencia interna que ofrece la masa de suelo por área unitaria para resistir la falla al deslizamiento a lo largo de cualquier plano dentro de él.

El esfuerzo cortante puede ser determinado de muchas maneras, algunos de los ensayos más comunes inclinadas son la veleta (ASTM D 4648), ensayos de penetración estándar - SPT (ASTM D 1586), así como algunos otros tipos de penetrómetros, los cuales en su mayoría no evitan los problemas asociados con la alteración de la muestra debido a su extracción en el campo, sin dejar de lado que ofrecen información sumamente importante. Sin embargo, muchos de esos métodos determinan la resistencia al cortante indirectamente a través de correlaciones. Por otra parte, en el laboratorio existe una serie de ensayos que usualmente se realizan dentro del ámbito de la ingeniería para evaluar las propiedades de resistencia de cada material que conforma el subsuelo. Entre estos se pueden citar la resistencia a la compresión uniaxial (ASTM D 2166), corte directo (ASTM D 3080 y ASTM D 6528) y los ensayos de compresión triaxial (ASTM D 4767 y ASTM D 2850).

\section{Criterios de Falla Mohr-Coulomb}

Mohr (1900) presentó una teoría sobre la ruptura de los materiales. Dicha teoría afirma que un material falla debido a una combinación crítica de esfuerzo normal y esfuerzo cortante, y no solo por la presencia de un esfuerzo máximo normal o bien de un esfuerzo máximo cortante. Por lo cual, la relación entre un esfuerzo normal y un esfuerzo cortante sobre un plano de falla se expresa en la Ecuación 1.

$$
\tau_{f}=f(\sigma)
$$

Donde:

$$
\begin{aligned}
& \tau_{f}=\text { esfuerzo cortante sobre el plano de falla } \\
& \sigma=\text { esfuerzo normal sobre el plano de falla }
\end{aligned}
$$

La envolvente de falla definida por la ecuación es una línea curva. Para la mayoría de los problemas de mecánica de suelos, es suficiente aproximar el esfuerzo cortante sobre el plano de falla como una función lineal del esfuerzo normal [9], lo cual se conoce como el Criterio de falla Mohr-Coulomb como se presenta en la Ecuación 2.

Donde:

$$
\tau_{f}=c+\sigma \tan \phi
$$

$$
\begin{aligned}
& C=\text { cohesión } \\
& \phi=\text { ángulo de fricción interna }
\end{aligned}
$$

En la Figura 1 se observa, que si el esfuerzo normal y el esfuerzo cortante sobre un plano en una masa de suelo son tales que son representados por el punto $A$, entonces no ocurrirá una falla cortante a lo largo de ese plano. Si el esfuerzo normal y el esfuerzo cortante sobre un plano son representados por el punto $B$ (sobre la envolvente de falla), entonces ocurrirá una falla de cortante a lo largo de ese plano. Un estado de esfuerzo ubicado en el punto $C$ no existe, porque queda por arriba de la envolvente de falla y la falla cortante ya habría ocurrido en el suelo.

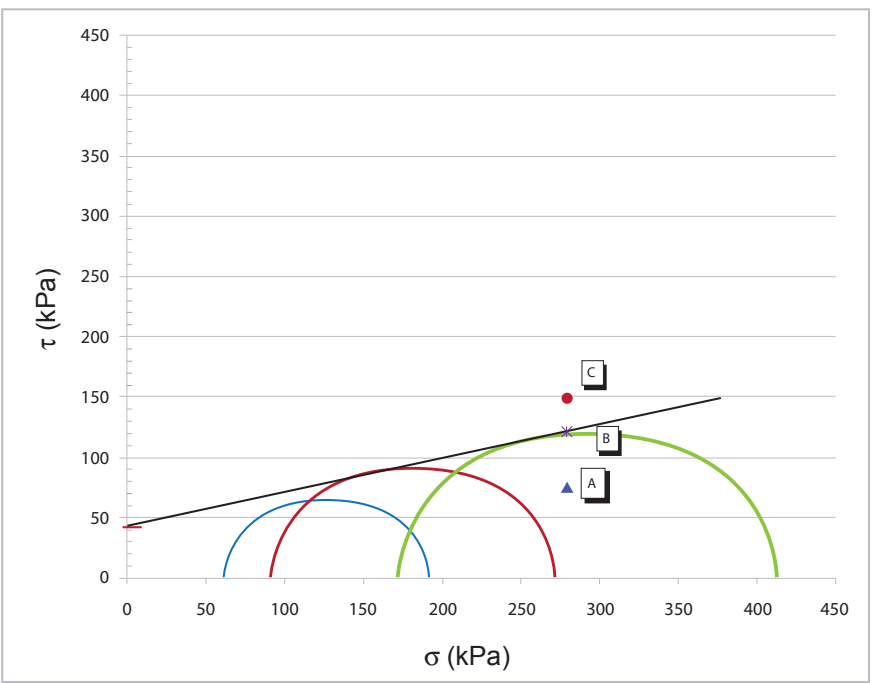

Figura 1. Envolvente de falla de Mohr

\section{Prueba Triaxial}

La prueba de ensayo triaxial es uno de los métodos más confiables para determinar los parámetros de la resistencia al cortante.

En un ensayo triaxial, un espécimen cilíndrico de suelo es revestido con una membrana de látex dentro de una cámara a presión. La parte superior e inferior de la muestra tiene discos porosos, los cuales se conectan al sistema de drenaje para saturar o drenar el espécimen. En estas pruebas se pueden variar las presiones actuantes en tres direcciones ortogonales sobre el espécimen de suelo, efectuando mediciones sobre sus características mecánicas en forma completa. Los especímenes usualmente están sujetos a presiones laterales de un líquido, generalmente agua.

El agua de la cámara puede adquirir cualquier presión deseada por la acción de un compresor comunicado con ella. La carga axial se transmite al espécimen por medio de un vástago que atraviesa la parte superior de la cámara. 
La presión que se ejerce con el agua que llena la cámara es hidrostática y produce por lo tanto, esfuerzos principales sobre el espécimen, iguales en todas las direcciones, tanto lateral como axialmente. En las bases del espécimen actuará además de la presión del agua, el efecto transmitido por el vástago de la cámara desde el exterior.

Es usual llamar $\sigma_{1}, \sigma_{2}$ y $\sigma_{3}$ a los esfuerzos principales mayor, intermedio y mínimo, respectivamente. En una prueba de compresión, la presión axial siempre es el esfuerzo principal mayor, $\sigma_{1}$; los esfuerzos intermedios y menor son iguales $\left(\sigma_{2}=\sigma_{3}\right)$ y son iguales a la presión lateral.

\section{Tipos de pruebas triaxiales}

\subsection{Prueba lenta - Prueba con consolidación y con drenaje (CD)}

La característica fundamental de la prueba es que los esfuerzos aplicados al espécimen son efectivos. Primeramente se aplica al suelo una presión hidrostática, manteniendo abierta la válvula de comunicación con la bureta y dejando transcurrir el tiempo necesario para que haya consolidación completa bajo la presión actuante. Cuando el equilibrio estático interno se haya restablecido, todas las fuerzas exteriores estarán actuando sobre la fase sólida del suelo, es decir, producen esfuerzos efectivos, en tanto que los esfuerzos neutrales en el agua corresponden a la condición hidrostática. La muestra se lleva a la falla a continuación aplicando la carga axial en pequeños incrementos, cada uno de los cuales se mantiene el tiempo necesario para que la presión en el agua, en exceso de la hidrostática, se reduzca a cero.

Los ensayos consolidados drenados se utilizan esencialmente en suelos granulares (arenas), sin embargo, se puede aplicar en suelos finos, pero los ensayos requieren tiempos prolongados del orden de semanas.

\subsection{Prueba rápida - Prueba con consolidación y sin drenaje (CU)}

En este tipo de prueba, el espécimen se consolida primeramente bajo la presión hidrostática; así el esfuerzo llega a ser efectivo, actuando sobre la fase sólida del suelo. En seguida, la muestra se lleva a la falla por un rápido incremento de la carga axial, de manera que no se permita cambio de volumen. El hecho esencial de este tipo de prueba es el no permitir ninguna consolidación adicional durante el periodo de falla, de aplicación de la carga axial. Esto se logra fácilmente en una cámara de compresión triaxial cerrando la válvula de salida de las piedras porosas a la bureta.
En la segunda etapa de una prueba rápida consolidada podría pensarse que todo el esfuerzo desviador fuera tomado por el agua de los vacíos del suelo en forma de presión neutral, ello no ocurre así y se sabe que parte de esa presión axial es tomada por la fase sólida del suelo, sin que hasta la fecha, se hayan dilucidado por completo ni la distribución de esfuerzos, ni las razones que lo gobiernan. De hecho no hay ninguna razón en principio para que el esfuerzo desviador sea íntegramente tomado por el agua en forma de presión neutral, si la muestra estuviese lateralmente confinada, como el caso de una prueba de consolidación.

El ensayo CU (consolidado-no drenado) se realiza generalmente con medición de la presión de poros o neutra con el fin de determinar los parámetros de "C" y " $\phi$ " en términos de esfuerzos totales y esfuerzos efectivos.

\subsection{Prueba rápida - Prueba sin consolidación y sin drenaje (UU)}

En este tipo de prueba no se permite en ninguna etapa la consolidación de la muestra. La válvula de comunicación entre el espécimen y la bureta permanece siempre cerrada impidiendo el drenaje. En primer lugar, se aplica al espécimen una presión hidrostática y de inmediato, se falla el suelo con la aplicación rápida de la carga axial. Los esfuerzos efectivos en esta prueba no se conocen bien.

El ensayo UU es usualmente llevado a cabo sobre especímenes de arcilla, enmarcando la realización del ensayo dentro del concepto de resistencia para suelos cohesivos saturados, en donde se expresan los resultados en términos de esfuerzos totales. La envolvente de falla para los criterios de Mohr del esfuerzo total se convierte en una línea horizontal, con una condición de $\phi=0^{\circ}$ (ángulo de fricción) y $\tau_{f}=C_{u}$, siendo $C_{u}$ la resistencia al cortante no drenada, la cual es igual al radio de los círculos de Mohr.

\section{Máquina triaxial - Laboratorio de Geotecnia - LanammeUCR}

\subsection{Componentes de la máquina triaxial}

Con el fin de realizar los diversos ensayos triaxiales, un equipo triaxial completo requiere diferentes elementos que lleven a cabo la adquisición de datos, aplicación de carga, medición de cambios de volumen, saturación de especímenes, entre otras funciones. A continuación se caracterizan de forma breve cada uno de ellos y la función que cumplen. 
Es el sistema conformado por válvulas y reguladores mediante los cuales se administra el flujo de aire y agua desaireada entre los equipos para la realización del ensayo triaxial. Cada panel posee 3 válvulas de distribución, reguladores de aire y salidas de medición de presión para 3 presiones (ver Figura 2).

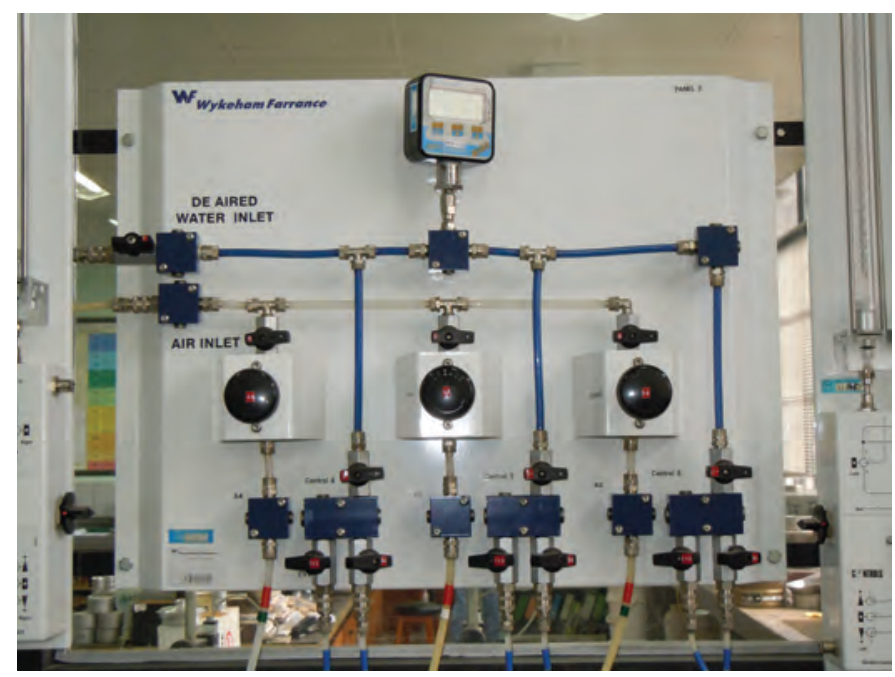

Figura 2. Panel de Control

\subsection{Equipo automático de cambio de volumen}

El equipo de cambio de volumen (aparato) realiza su función comprimiendo un pistón sellado contra un dispositivo de precisión en la cámara de calibración, de tal forma que un movimiento lineal del pistón es exactamente proporcional al cambio de volumen de agua que se da en la cámara de calibración (ver Figura 3). El pistón está conectado a un medio de medición externo, un transductor de desplazamiento lineal, adecuado para el sistema de adquisición de datos permitiendo que los cambios de volumen de la muestra sean desplegados y registrados directamente en centímetros cúbicos.

La unidad está conectada a un panel de control con cambio de volumen y regulador de flujo (by pass valves) usados para medir la saturación y cambios de volumen mayores a $100 \mathrm{cc}$.
Las especificaciones técnicas de este dispositivo son:

\begin{tabular}{|c|c|}
\hline Pistón & $40.15 \mathrm{~cm}^{2}$ \\
\hline Distancia de recorrido & $25 \mathrm{~mm}$ \\
\hline Capacidad de operación & $100 \mathrm{~cm}^{3}$ \\
\hline Precisión & $\pm 0.05 \mathrm{~cm}^{3}$ \\
\hline Dimensiones generales & $225 \times 230 \times 390 \mathrm{~mm}(\mathrm{LxDxH})$ \\
\hline Entrada de transductor mayor a & $12 \mathrm{Vdc}$ \\
\hline Carrera del transductor & $27 \mathrm{~mm}$ \\
\hline
\end{tabular}

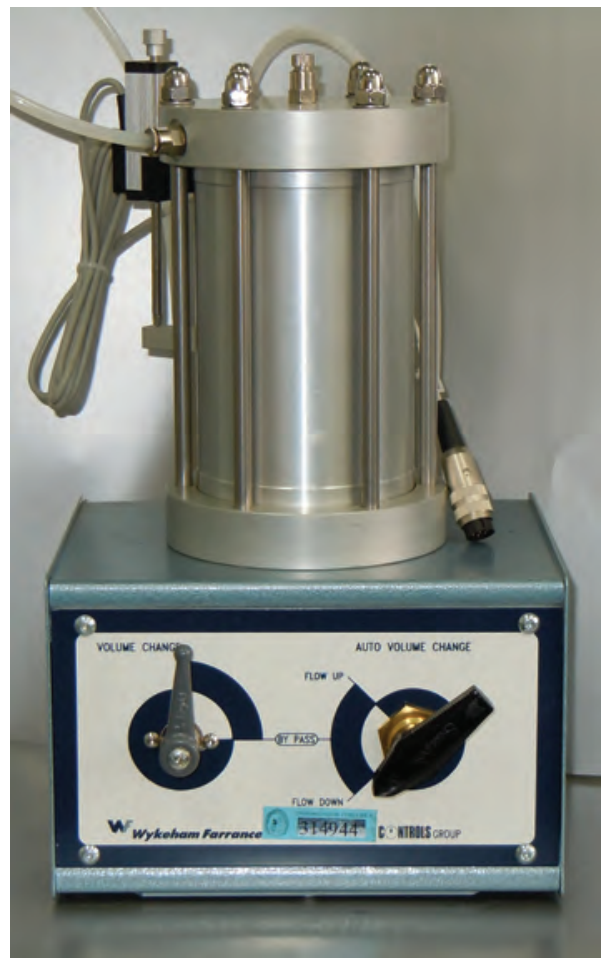

Figura 3. Dispositivo de cambio de volumen

\subsection{Prensa Triaxial}

La prensa triaxial consiste en un marco de dos columnas con una viga transversal móvil (marco de carga) y una base que contiene la unidad de empuje mecánico, el motor eléctrico, los componentes electrónicos y los controles (ver Figura 4). La acción de carga es realizada por un motor (stepper motor) de alta resolución. La unidad de sincronización que maneja el motor es controlada por un microprocesador. Mediante este microprocesador es posible obtener un desplazamiento predeterminado de la unidad de carga (empuje), constante durante el ensayo, cualquiera que sea la fuerza de resistencia. 
La velocidad y la dirección se preestablecen a través de controles localizados en el panel frontal.

Las especificaciones técnicas de este dispositivo se encuentran a continuación:

\begin{tabular}{|c|c|}
\hline $\begin{array}{c}\text { Mínima velocidad de } \\
\text { desplazamiento (ensayo) }\end{array}$ & $1 \times 10^{-5} \mathrm{~mm} / \mathrm{min}$ \\
\hline $\begin{array}{c}\text { Máxima velocidad de } \\
\text { desplazamiento (ensayo) }\end{array}$ & $10 \mathrm{~mm} / \mathrm{min}$ \\
\hline Velocidad de aproximación rápida & $25 \mathrm{~mm} / \mathrm{min}$ \\
\hline $\begin{array}{l}\text { Capacidad máxima de ensayo } \\
\text { (Carga) }\end{array}$ & $50 \mathrm{kN}$ \\
\hline Sistema de limitación de movimiento & $\begin{array}{c}2 \text { micro interruptores más dos micro } \\
\text { interruptores digitales }\end{array}$ \\
\hline Motor & $\begin{array}{l}\text { De } 5 \text { fases y } 1000 \text { impulsos (round } \\
\text { stepper motor) }\end{array}$ \\
\hline Espacio horizontal máximo & $380 \mathrm{~mm}$ \\
\hline Espacio vertical máximo & $850 \mathrm{~mm}$ \\
\hline Dimensiones generales & $500 \times 273 \times 1266 \mathrm{~mm}(\mathrm{LxDxH})$ \\
\hline
\end{tabular}

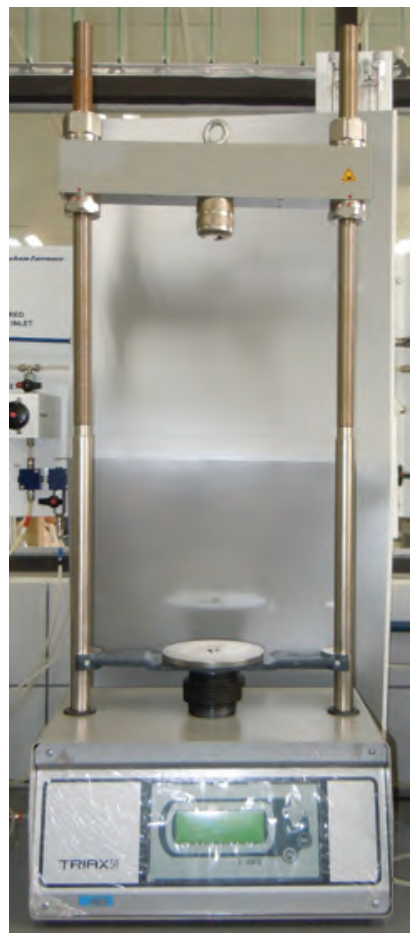

Figura 4. Prensa Triaxial

\subsection{Blader}

Es una cámara constituida por un cilindro de bandas de plexiglass, una placa base, una placa superior y una membrana de hule que trabaja como interface aire/agua (ver

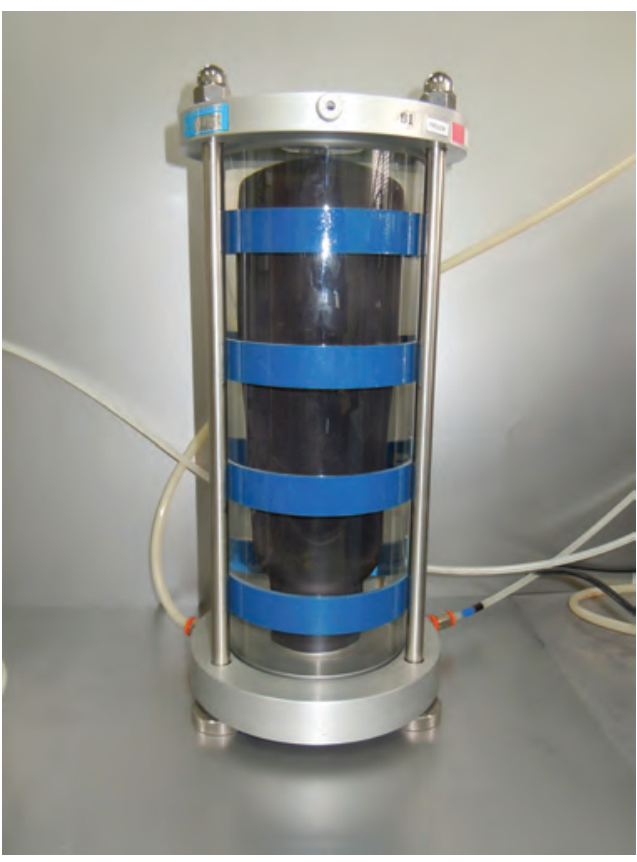

Figura 5. Blader

Figura 5). La membrana de hule está fijada a la placa base mediante un collar plástico.

El blader es el encargado de transferir la presión del aire al agua, de forma inmediata, evitando que el aire comience a disolverse. La presión máxima de funcionamiento del blader es de $1000 \mathrm{kPa}$.

\subsection{Cámara triaxial}

Está conformada por una cámara de bandas de metacrilato que permiten una presión máxima de funcionamiento de

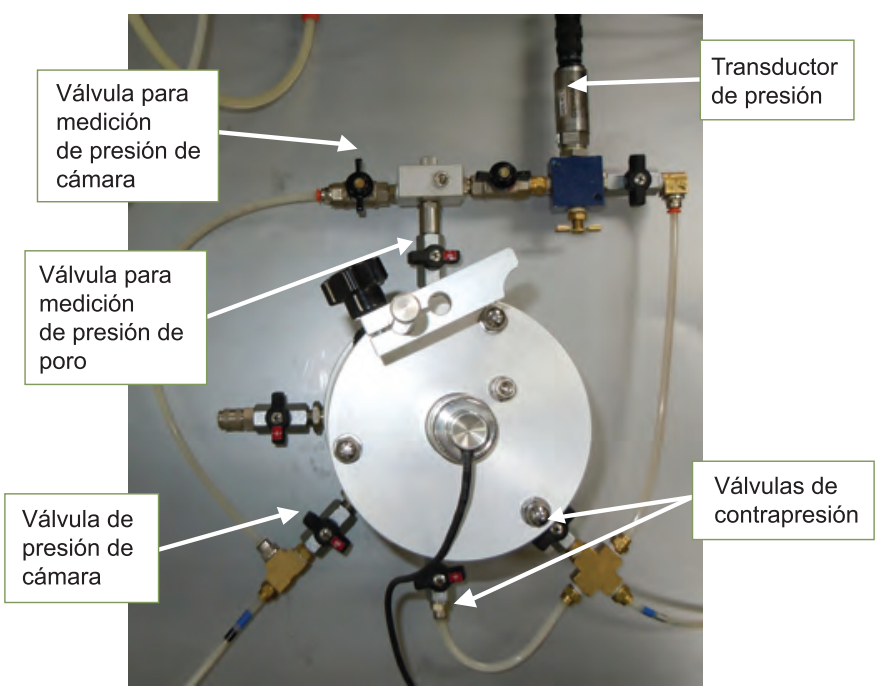

Figura 6. Cámara Triaxial 
$2000 \mathrm{KPa}$, una base con cinco conexiones, de las cuales dos son para presión de poro, dos para contrapresión y una para presión de cámara. A su vez cuenta con un pistón de carga instalado mediante un sistema de baja fricción. Es en la cámara triaxial en donde se somete al espécimen a las condiciones específicas de esfuerzos de los ensayos (ver Figura 6).

\subsection{Transductores}

Los transductores se encargan de transformar una señal eléctrica en una magnitud física, la cual, en este caso, es enviada al dataloger para así poder registrar tanto las presiones a las que está sometido el espécimen como las deformaciones que sufre durante el ensayo.

Para la automatización del ensayo triaxial se requieren transductores lineales, los cuales miden desplazamientos (ver Figura $7^{\mathrm{a}}$ ), y transductores de presión (ver Figura 7b) cuyas características se mencionan a continuación:

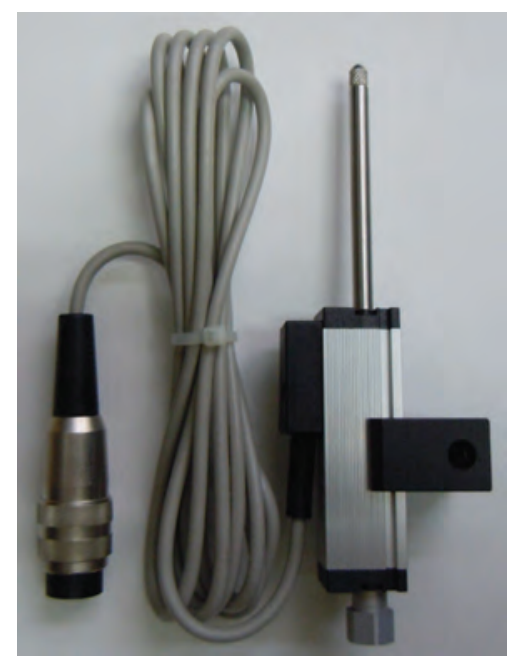

a) LVDT

b) Transductor de presión

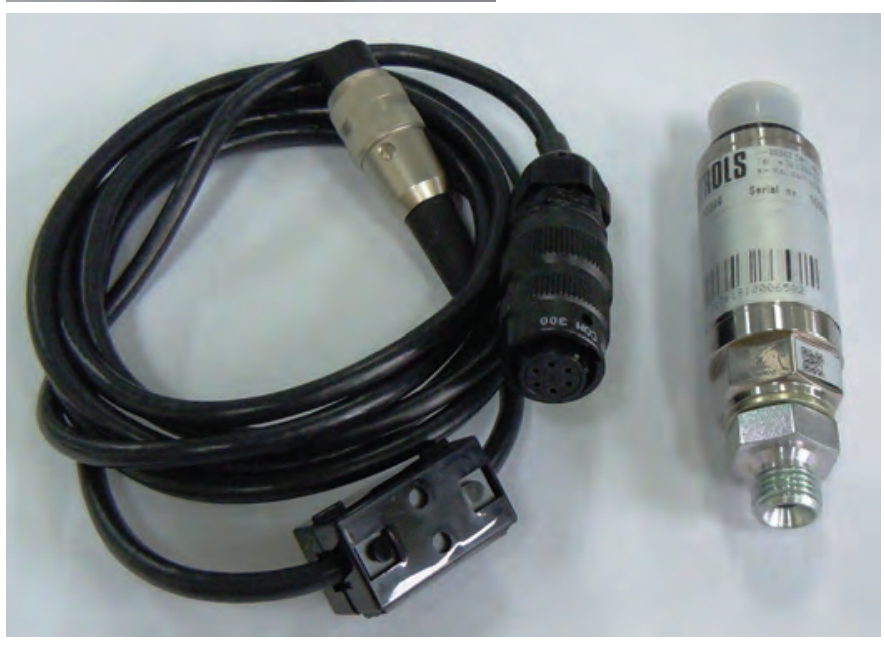

Figura 7. LVDT y Transductores
Transformador de diferencial lineal variable (LVDT)

-Rango de medición

$10 \mathrm{~mm}$

-Resistencia nominal

$1 \mathrm{Kohm}$

-Fuente de voltaje

10 VDc máxima

\section{Transductor de presión}

-Presión máxima de medición

10 bar

-Excitación

$10 \mathrm{~V} \mathrm{dc/ac} \mathrm{RM}$

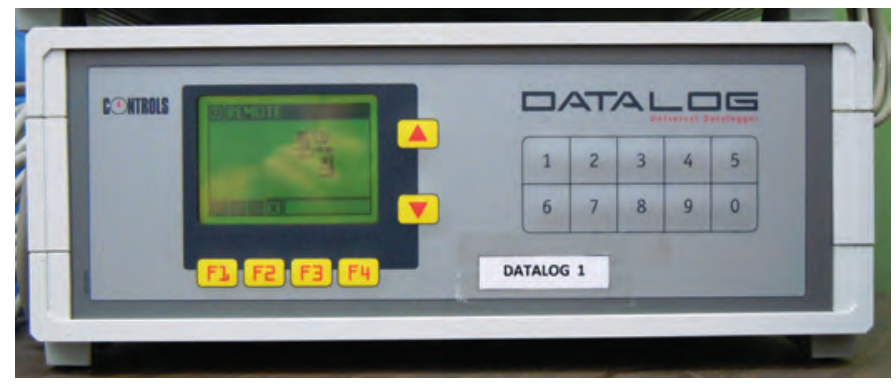

Figura 8. DATALOG

\subsection{Dataloger}

El DATALOG, es una nueva serie de los Universal Data Loggers, con 8 canales de micro-procesamiento para la adquisición de datos provenientes de las señales eléctricas de los transductores (ver Figura 8).

Este se encarga de registrar datos en tiempo real y almacenarlos, brindando la posibilidad de transferir dichos registros a la computadora. Mediante este, también se realiza la calibración de los transductores, lo cual se lleva a cabo mediante comparación con mediciones de instrumentos de precisión.

Por medio del Datalog se pueden realizar dos tipos de calibración:

\section{-Calibración lineal}

Usada generalmente, esta asume una relación lineal entre la señal de salida eléctrica (o digital) del transductor y la fuerza aplicada (presión, desplazamiento, temperatura, etc.). En este caso se establece un factor de calibración para mostrar el registro o los datos directamente en la unidad física seleccionada.

\section{- Calibración polinomial}

En esta la relación, carga aplicada no es directamente proporcional a la salida eléctrica (o digital) pero puede ser expresada mediante una ecuación polinomial de segundo o tercer grado. 
La unidad está construida de un contenedor plástico con una cubierta metálica interior, a prueba de perturbación electromagnética de alta frecuencia. Está equipado con un teclado de policarbonato anti-ralladuras con 16 teclas y una pantalla gráfica monocromática.

En la parte posterior del panel se localizan:

-8 conectores hembra para conectar los transductores.

-Puerto serie RS232.

-Puerto serie RS485/CAN.

-Fuente de alimentación.

-Interruptor principal y conectores auxiliares.

\subsection{Equipo Secundario}

-Bomba de vacío: se emplea para llenar el tanque de almacenamiento de agua destilada así como para eliminar el aire, tanto el del tanque como el que se encuentra disuelto en el agua.

-Compresor: este suministra aire a presión al sistema.

-Depósito auxiliar de aire: como su nombre lo indica, almacena aire a presión. Su capacidad es de 50 L y soporta una presión máxima de 14.5 bar.

-Tanque de almacenamiento de agua destilada.

-Manómetro digital de presión.

-Buretas de cambio de volumen.

\section{Ejecución de ensayos}

Se ha experimentado con el equipo en la realización de ensayos. A continuación se presenta una serie de resultados, de un ensayo UU (no consolidado - no drenado) ejecutado a un suelo MH (limo de alta plasticidad), color café claro con pequeñas vetas de roca color blancuzco, extraído de la zona de San Ramón de Alajuela.

Algunas de las propiedades del suelo se enumeran en la Tabla 1.

Tabla 1. Propiedades del suelo ensayado

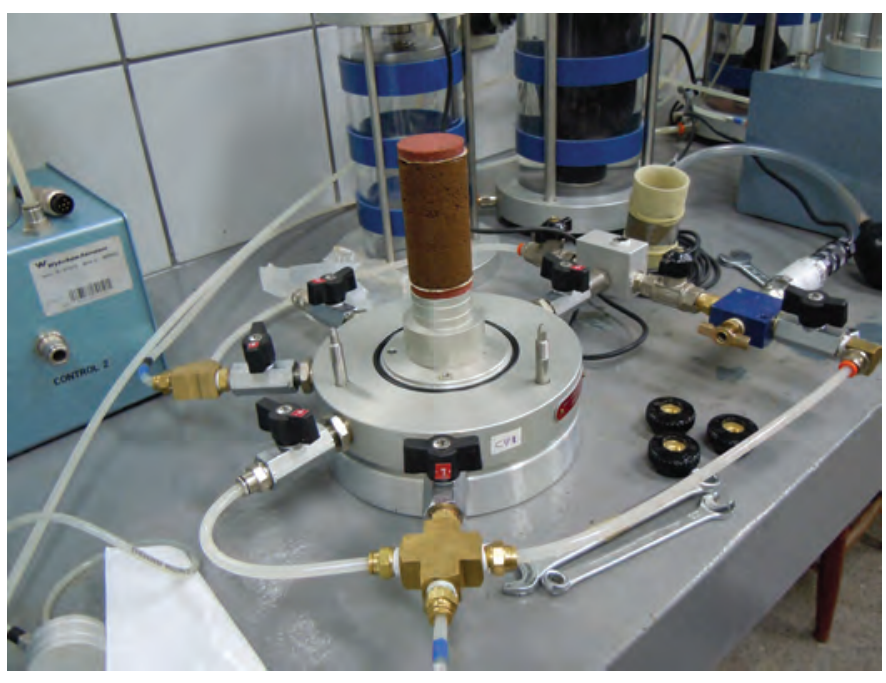

Figura 9. Montaje de la pastilla

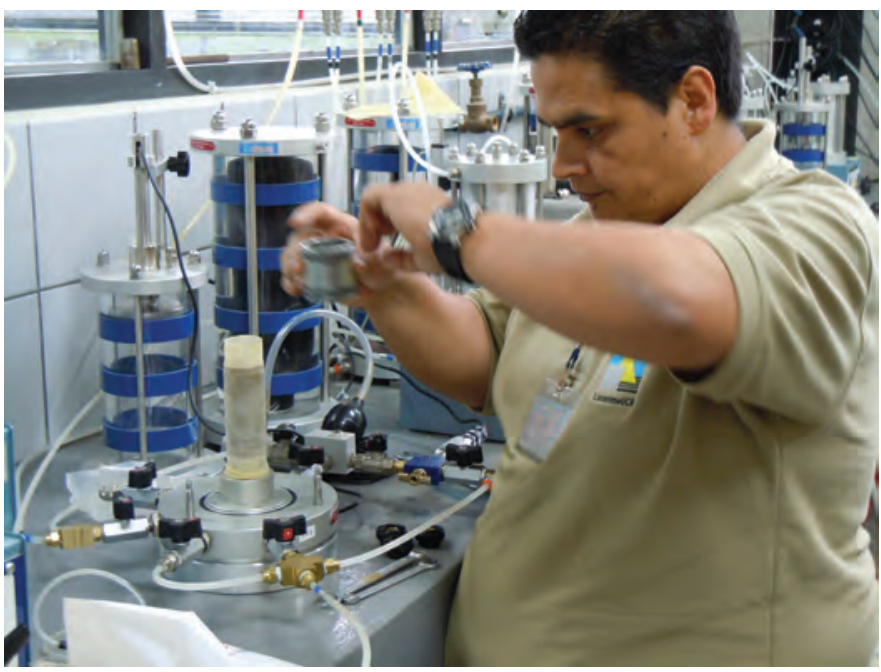

Figura 10. Revestimiento de la pastilla con membrana

De la Figura 9 a la Figura 16 se muestra una secuencia de imágenes en donde se describe los pasos para la falla de los especímenes. Primero se muestra cómo el espécimen luego de ser moldeado o remoldeado, debe ser colocado en la cámara y luego es recubierto con la membrana de latex. Posteriormente, se coloca la cámara con su debida celda de carga, luego se inunda la muestra y de este modo se le suministra la presión de cámara respectiva, según sea el rango de cargas que se deban aplicar. Se muestra a su vez la cámara, inmersa en el marco de falla, el cual es capaz de aplicarle una velocidad de carga constante durante la falla.

\begin{tabular}{|c|c|c|c|c|c|c|c|}
\hline Límites de Atterberg & $G_{s}$ & Porentaje pasando No. 200 & Clasificación & $\begin{array}{l}\text { Humedad natural } \\
(\%)\end{array}$ & $\begin{array}{c}\text { Densidades } \\
\text { húmedas }\left(\mathbf{g} / \mathrm{cm}^{3}\right)\end{array}$ & $\begin{array}{c}\text { Humedad } \\
\text { Especímen (\%) }\end{array}$ & Saturación (\%) \\
\hline $\mathrm{LL}=84$ & & & & & & & \\
\hline $\begin{array}{l}\mathrm{LP}=61 \\
\mathrm{IP}=23\end{array}$ & 2.823 & 90.3 & $\mathrm{MH}$ & 20.8 & $1.47-1.52$ & $58.4-63.6$ & 100 \\
\hline
\end{tabular}

Simbología:

MH: Limos de alta plasticidad.

Gs: Gravedad específica de los sólidos del suelo. 


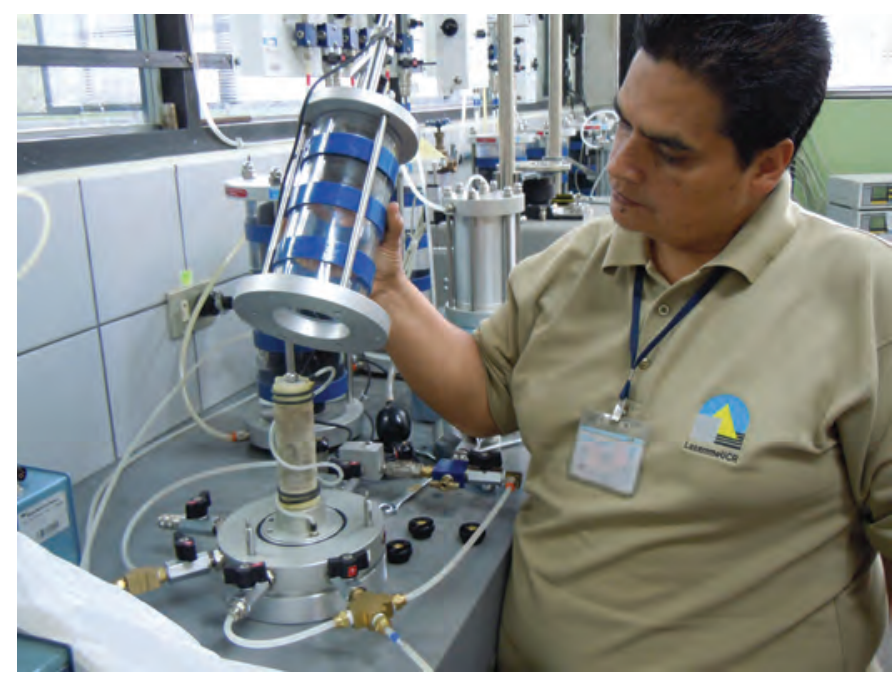

Figura 11. Colocación de cámara

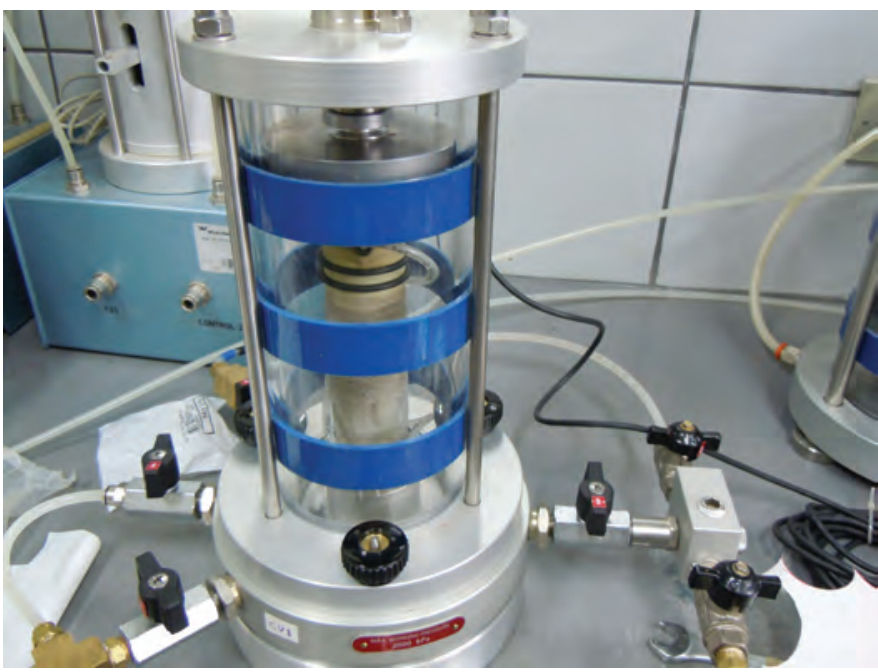

Figura 12. Llenado de cámara con lucita

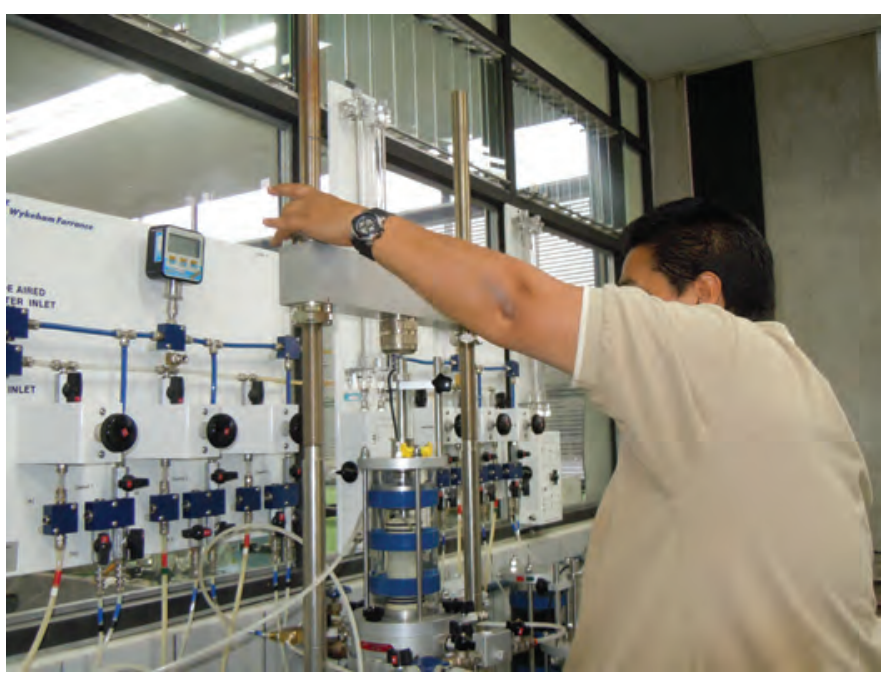

Figura 13. Montaje en la prensa de carga

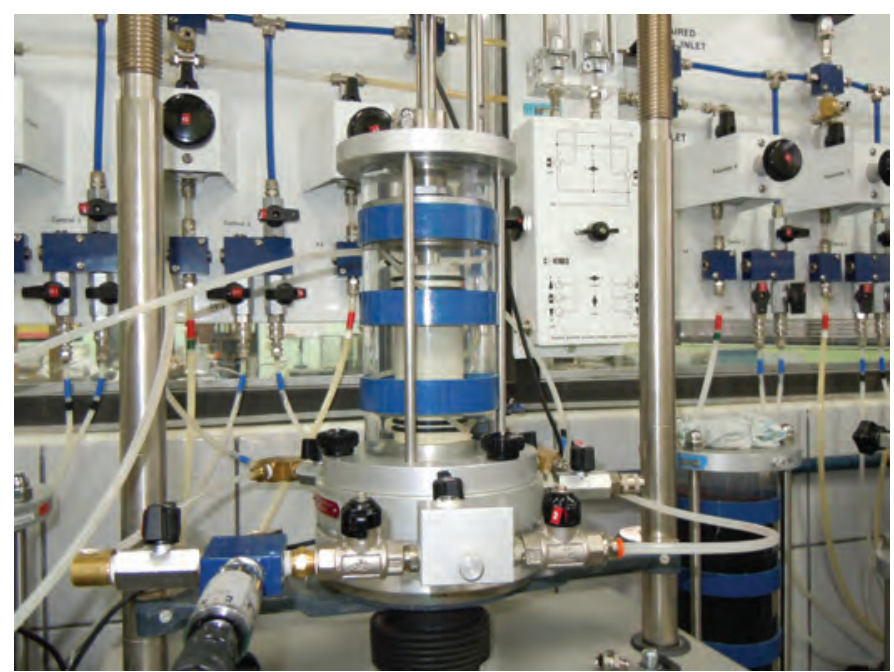

Figura 14. Falla del espécimen

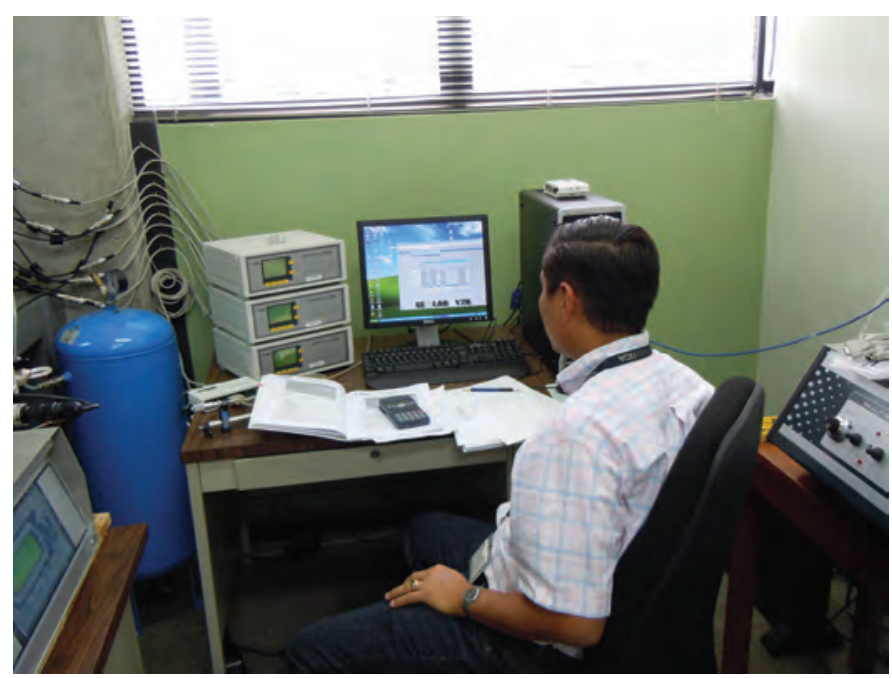

Figura 15. Procesamiento y captura de datos

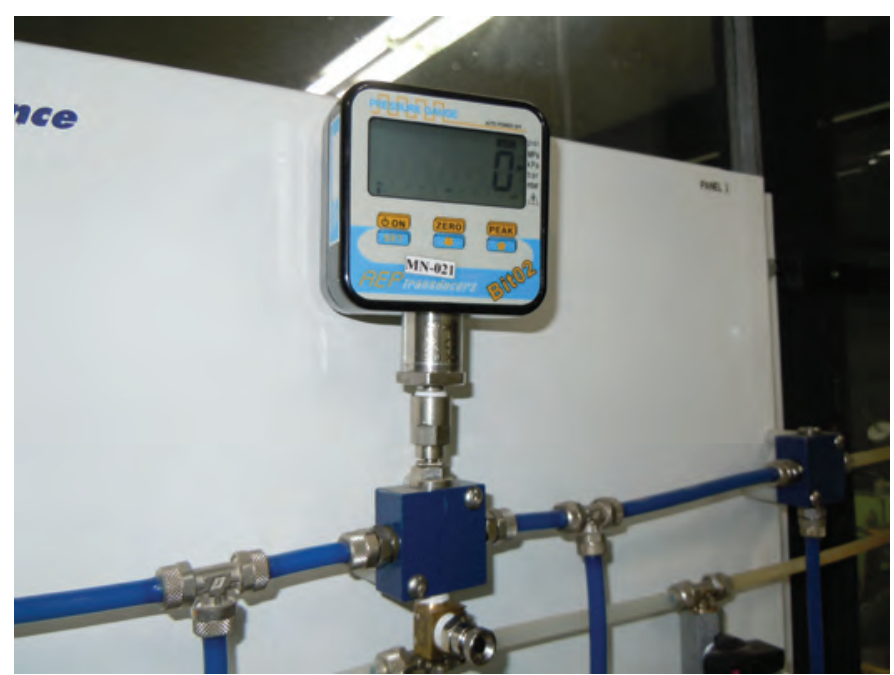

Figura 16. Lecturas de presiones y contrapresiones 
Para una gran mayoría de ensayos triaxiales es necesario como fase previa a la realización de una prueba, la saturación del espécimen, esto se puede lograr mediante la aplicación de presiones y contrapresiones a la muestra, así como con variaciones en las mismas, donde la saturación se genere en el espécimen con la menor perturbación. En la Figura 17 se logra evidenciar dicho proceso de saturación, reflejado en las lecturas de presión de poros. En dicha figura se representa como al generar una diferencia de presiones, la presión de poros leída logra igualar dicha diferencia y manteniéndose constante en el tiempo, leyéndose por tanto un valor de $B$ cercano a 1,002. ( $B=1,002$ - Skempton).

En la Figura 18, se logra apreciar un gráfico de fuerza en función del tiempo. La fuerza reflejada en dicho gráfico es la fuerza producto de la falla que se genera al someter

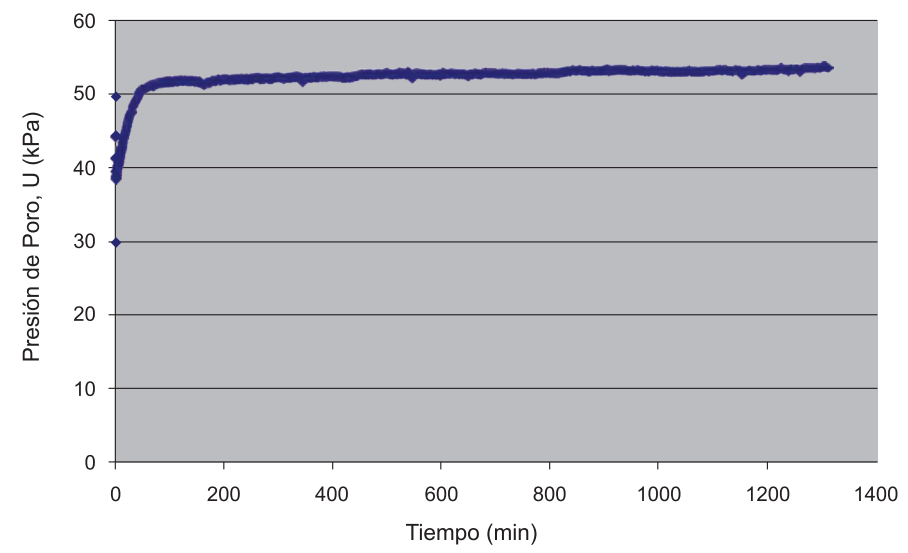

Figura 17. Corroboración de la saturación (Parámetro B Skempton)

el espécimen a un esfuerzo desviador a una velocidad constante, medido en el tiempo, donde se evidencia su incremento a lo largo del desempeño de la misma, un punto de máxima resistencia y posteriormente la caída en la carga, producto ya de la falla del espécimen. Esto se observa en el caso del tercer espécimen, el cual fue sometido a un esfuerzo confinante de $200 \mathrm{kPa}\left(\sigma_{3}=200 \mathrm{kPa}\right)$.

En las Figuras 19 y 20, se puede observar algunas de las etapas propias del programa de adquisición y procesamiento de datos. En la figura 19 específicamente se muestra la verificación de la adecuada lectura de los instrumentos. La figura 20 , evidencia los segundos previos al inicio de la falla, en donde el ejecutante se debe asegurar de "poner en cero" todos los respectivos instrumentos.

Por último, la Figura 21, muestra el resultado final, resumen de los esfuerzos obtenidos de la aplicación de un ensayo

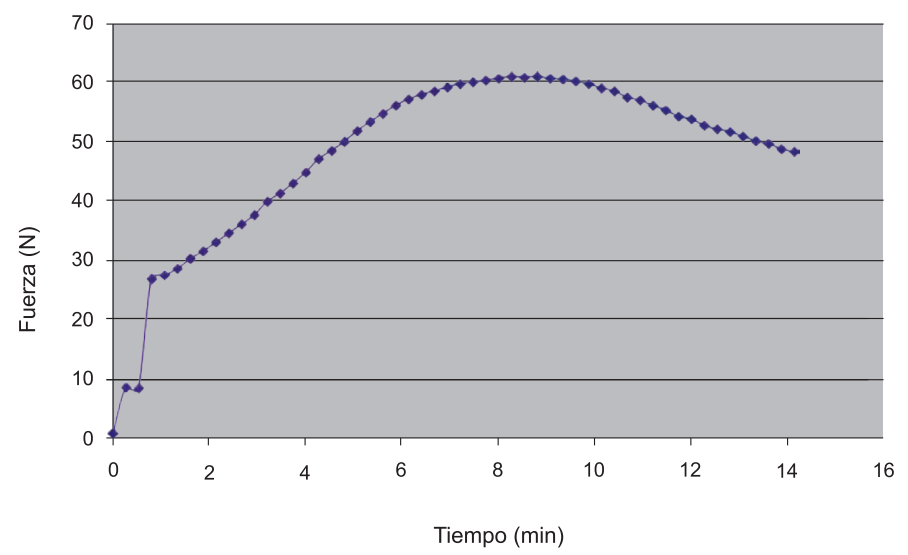

Figura 18. Fase rotura espécimen $3(\sigma 3=200 \mathrm{kPa})$

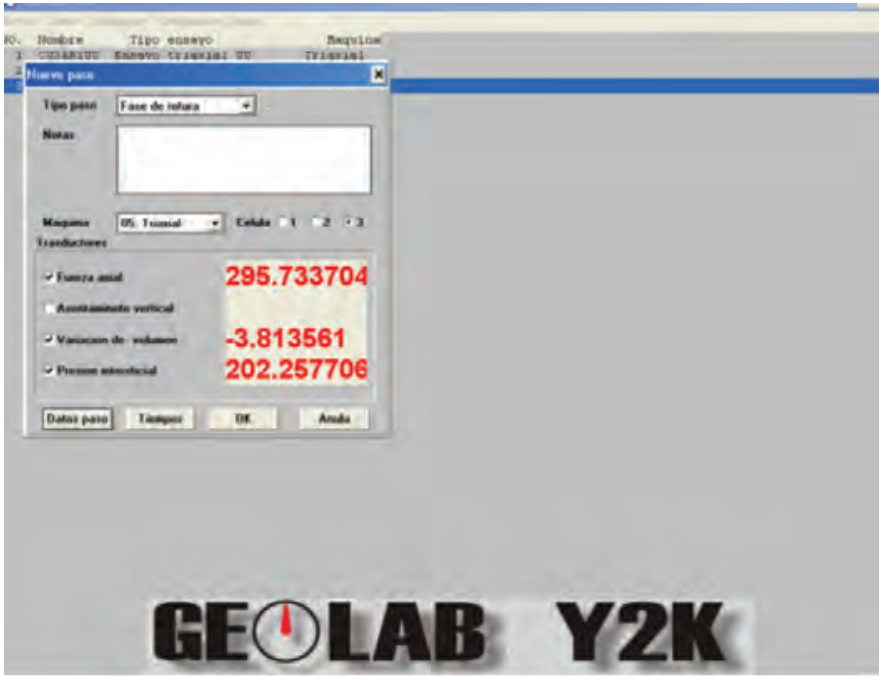

Figura 19. Fase rotura-lectura de instrumentos, cámara 3

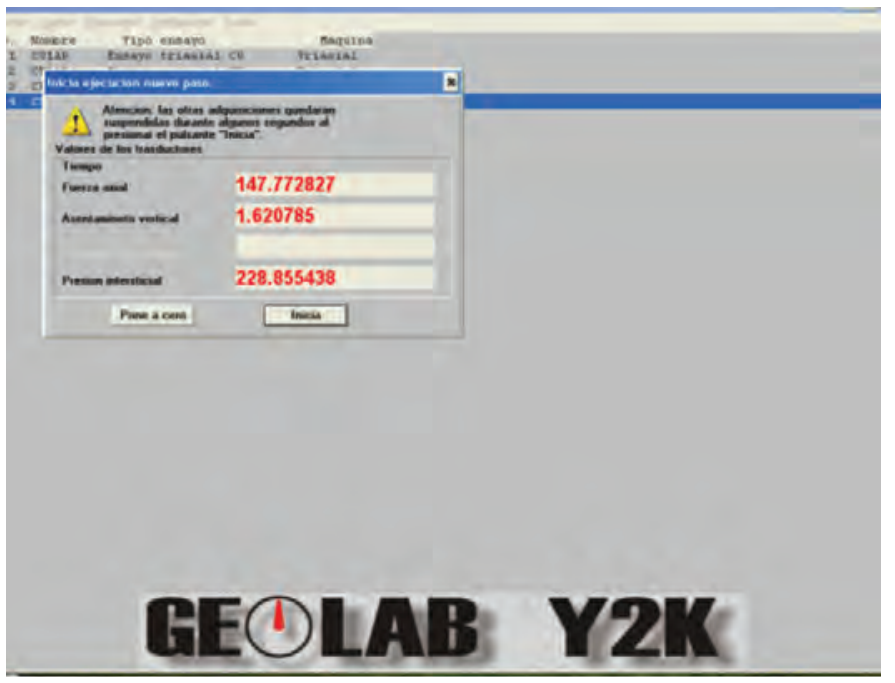

Figura 20. Fase rotura-cero de los instrumentos 


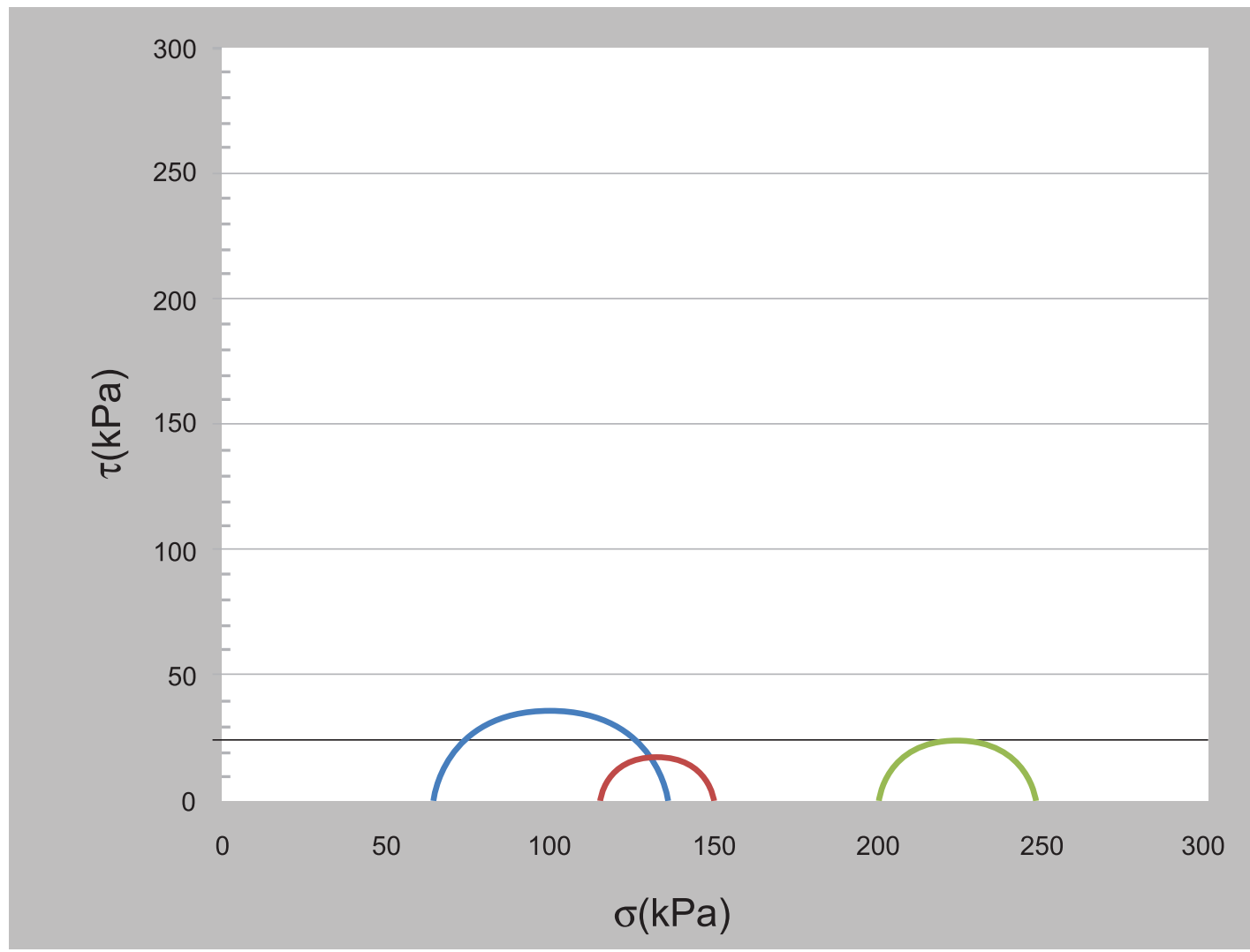

Figura 21. Resumen de resultados

UU, dando como parámetro final una cohesión no drenada. (Ángulo de fricción $\phi=0^{\circ}$, cohesión no drenada $\mathrm{Cu} \approx 25$ $\mathrm{kPa})$.

\section{Conclusiones y recomendaciones}

La investigación de suelos es la base para un buen diseño de una obra civil. Cuando se envían muestras de un suelo a un laboratorio para ser ensayadas, dichos resultados son a su vez la base para el análisis y toma de decisiones. Sin embargo, dichos resultados de laboratorio son útiles y contribuyen a decisiones acertadas sólo si reflejan las condiciones que representan adecuadamente la superficie de suelo que se requiere intervenir.

Ahora, por su parte los resultados emitidos por un laboratorio tienen que arrojar resultados confiables, producto de la ejecución de ensayos debidamente normados y con los equipos adecuados.

Antes de la realización de los ensayos triaxiales, es importante tener en consideración algunos aspectos importantes como paso fundamental, tener las tuberías que conducen el agua a cada uno de los diferentes componentes (equipos de cambio de volumen, blader de presión y contrapresión, etc.) debidamente purgados, es decir, que se hayan evacuado todas las burbujas de aire, dentro del sistema, para que no se generen vacíos que afecten tanto la muestra como que se generen lecturas de los instrumentos erróneas. Incluso para algunos instrumentos es conveniente la aplicación de presiones iniciales para así ayudar a eliminar la presencia de burbujas.

Es necesario asegurarse que los instrumentos de medición se encuentren debidamente ubicados en el respectivo Datalog, así como en su respectivo canal. Verificar que las unidades de medición sean las correctas y que la ecuación de ajuste sea la correspondiente.

Si la prueba lo amerita, se requerirá como etapa inicial, la verificación de la saturación del espécimen. Es importante entonces tener presente las presiones aplicadas, tanto la presión como la contrapresión, parámetros que se ven reflejados directamente en la presión de poros de la muestra, esto para no incurrir en errores en el cálculo del parámetro B de Skempton. Es conveniente considerar y valorar la opción de la saturación de los especímenes en pruebas como las no consolidadas no drenadas, especialmente si se está al frente de suelos tropicales. 
Es importante tener especial cuidado de concentrar y medir las presiones requeridas, ya sean presiones de cámara como contrapresiones, en sus respectivos componentes (blader) y luego transferirlas a los especímenes de forma gradual, para que la muestra se altere lo menos posible.

Finalmente una manipulación adecuada de las muestras, mínimo contacto con las mismas, tanto en su moldeo/ remoldeo como en su colocación en las cámaras, y una prevención extrema en la pérdida de humedad de los especímenes, son fundamentales como punto de partida para un óptimo desempeño del ensayo a ejecutar.

\section{Referencias}

1. American Society for Testing and Materials, ASTM D - 1586 -08a, Standard Test Method for Standard Penetration Test (SPT) and Split-Barrel Sampling of Soils, EEUU.

2. American Society for Testing and Materials, ASTM D - 2166 -06, Standard Test Method for Unconfined Compressive Strength of Cohesive Soils, EEUU.

3. American Society for Testing and Materials, ASTM D - 2850 -03a(2007), Standard Test Method for Unconsolidated-Undrained Triaxial Compression Test on Cohesive Soils, EEUU.

4. American Society for Testing and Materials, ASTM D - 308004, Standard Test Method for Direct Shear Test of Soils Under Consolidated Drained Conditions, EEUU.

5. American Society for Testing and Materials, ASTM D - 4648/ D4648M-10, Standard Test Method for Laboratory Miniature Vane Shear Test for Saturated Fine-Grained Clayey Soil, EEUU.
6. American Society for Testing and Materials, ASTM D 476711, Standard Test Method for Consolidated Undrained Triaxial Compression Test for Cohesive Soils, EEUU.

7. American Society for Testing and Materials, ASTM D - 652807, Standard Test Method for Consolidated Undrained Direct Simple Shear Testing of Cohesive Soils, EEUU.

8. Bardet, Jean Pierre. 1997. Experimental Soil Mechanics. California-EEUU: Prentice Hall.

9. Braja M., Das. 2001. Fundamentos de Ingeniería Geotécnica. México: Thomson Learning.

10. CONTROLS, Manual de instrucciones-Datalog 82-P0908/ A, 82-P0908/B, 30-T0601, Italia.

11. CONTROLS, Manual de instrucciones-Geo2000-New Software for data adquisition and procesing of Geotechnical Tests, Italia.

12. CONTROLS, Manual de instrucciones-Sistema automático de medición de variación de volumen, Italia.

13. Holtz., R. y Kovacs, W., 1981. An Introduction to Geotechnical Engineering. EEUU, Prentice-Hal International.

14. Juarez Badillo, E. y Rico Rodríguez. A, 1988. Mecánica de Suelos-Tomo 1-Funadamentos de Mecánica de Suelos. México: Editorial Limusa S.A DE CV.

15. Ortiz Mora, J. 2001. Comportamiento de una arena bajo carga monotónica en ensayos de compresión triaxial en condición no drenada. Tesis de Grado para obtener el grado de Licenciado en Ingeniería Civil. San Pedro-Costa Rica. 